\title{
Stationary Wavelet Transform for denoising Pulsed Thermography data: optimization of wavelet parameters for enhancing defects detection
}

\author{
by G. M. Revel*, E. Copertaro*, P. Chiariotti*, G. Pandarese*, A. D’Antuono*
}

* Università Politecnica delle Marche, DIISM, Via Brecce Bianche, 60131, Ancona, IT. e.copertaro@pm.univpm.it

\begin{abstract}
Innovative denoising techniques based on Stationary Wavelet Transform (SWT) have started being applied to Pulsed Thermography (PT) sequences, showing marked potentialities in improving defect detection. In this contribution, a SWT-based denoising procedure is performed on high and low resolution PT sequences. Samples under test are two composite panels with known defects. The denoising procedure undergoes an optimization step. An innovative criterion for selecting the optimal decomposition level in multi-scale SWT-based denoising is proposed. The approach is based on a comparison, in the wavelet domain, of the information content in the thermal image with noise propagated. The optimal wavelet basis is selected according to two performance indexes, respectively based on the probability distribution of the information content of the denoised frame, and on the Energy-to-Shannon Entropy ratio. After the optimization step, denoising is applied on the whole thermal sequence. The approximation coefficients at the optimal level are moved to the frequency domain, then low-pass filtered. Linear Minimum Mean Square Error (LMMSE) is applied to detail coefficients at the optimal level. Finally, Pulsed Phase Thermography (PPT) is performed. The performance of the optimized denoising method in improving the defect detection capability respect to the non-denoised case is quantified using the Contrast Noise Ratio (CNR) criterion.
\end{abstract}

\section{Introduction}

Stationary Wavelet Transform, also addressed as Undecimated Wavelet Transform, differs from the standard Discrete Wavelet Transform for the characteristic up-sampling of filters at various levels [1]. When applied to image denoising, this feature leads to the possibility of maintaining the original resolution, which would be lost otherwise. The advantage of preserving image resolution is evident when denoising aims at enhancing small details.

SWT-based denoising has been recently applied to PT results, in the field of Non Destructive Testing (NDT). Its impact on different resolution thermal images has been investigated in [2]. An interesting SWT-based denoising algorithm has been discussed in [3], where SWT is exploited in conjunction with noise filtering in the frequency domain. In [4], a LMMSE intra-scale and inter-scale model is applied to wavelet coefficients. Also, a criterion for selecting the optimal basis is presented. The approach is tested in image enhancement, showing remarkable performances with respect to other methods.

This paper presents an innovative criterion for selecting the optimal decomposition level in a SWT-based denoising algorithm applied to PT sequences. The approach consists in comparing, in the wavelet domain, the information content with the noise propagated into the wavelet coefficients. The optimal wavelet basis is selected according to a criterion based on the probability distribution of the information content of the denoised frame (the same criterion is presented and discussed in [4]) and to the Energy-to-Shannon-Entropy Ratio criterion [5]. Once the optimization step is concluded, the thermal images are denoised, then PPT is performed on the whole thermal sequence. The full diagnostic procedure is applied on thermal images acquired during PT tests performed on two types of composite panels. The first specimen is a Carbon Fiber Reinforced Polymer (CFRP) panel with Teflon $\circledast$ insertions simulating inclusion defects of different sizes and depths. The second sample is a Glass Reinforced Concrete (GRC) panel used in the construction sector as structural component, with flat holes on one side, simulating inclusions, cavities and fibers agglomerations. Indeed, the precast/prefabricated construction market has been growing as well the quality and the energy performance required to the panels, for this the development of inspection techniques aiming at locating defects/damages represents a hot topic in NDT. The EU project INSITER [6] (Intuitive Self-Inspection Techniques using Augmented Reality for construction, refurbishment and maintenance of energy-efficient buildings made of prefabricated components), for instance, is facing the issue of developing new inspection techniques for the assessment of the structural integrity of prefabricated panels.

The raw thermal images acquired during the PT tests are found to be affected by noise: detection of low- and high-depth defects is thus prevented. Results reported in the following will show how the use of the method proposed can drastically improve the defect detection capability of a post-processing technique as PPT. The CNR criterion is used for quantifying the improvement.

The paper is organized as follows: the measurement setup is described in Section 2; the denoising process and the optimization of the wavelet parameters are addressed in Section 3; Section 4 shows the performances of the denoising process in terms of improvements of the defect detection capability; Section 5 draws the main conclusions of the work. 


\subsection{1/qirt.2016.025}

\section{Measurement setup}

PT tests in reflection mode were performed on two specimens. The first is a CFRP panel $(280 \times 280 \times 4 \mathrm{~mm})$ with Teflon ${ }^{\circledR}$ insertions simulating inclusion defects of different sizes $(5 \times 5 \mathrm{~mm}, 10 \times 10 \mathrm{~mm}, 15 \times 15 \mathrm{~mm})$ at different depths $(0.3$ $\mathrm{mm}, 1 \mathrm{~mm}, 2 \mathrm{~mm}$ ), see Figure 1c. The second is a GRC panel presenting two flat holes (20 mm diameter) on one side, at different depths $(7 \mathrm{~mm}, 8.5 \mathrm{~mm})$ with respect to the defect-free surface, which coincides with the measurement surface, see Figure 1d. The holes were closed with an insulating material to simulate a barrier for the thermal transmittance (inclusion or cavity). The emissivity of the GRC panel is quite low (0.6), thus worsening the defects-background contrast.

The CFRP panel was excited with a pulsed heat load of $6 \mathrm{~kJ}$ provided on a $700 \mu$ s time interval, using two flash lamps. The temperature of the surface was monitored (acquisition rate: $30 \mathrm{~Hz}$; acquisition period: $40 \mathrm{~s}$ ) using a highresolution infrared camera (1024 x 768 uncooled micro-bolometric Focal Plane Array). In the second test, the GRC panel was heated for $60 \mathrm{~s}$ using halogen lamps with a total power of $1.5 \mathrm{~kW}$. The temperature of the defect-free surface was monitored (acquisition rate: $1 \mathrm{~Hz}$; acquisition period: $360 \mathrm{~s}$ ) using a different camera (320x240 uncooled micro-bolometric Focal Plane Array). The two experimental setups are shown in Figure 1a and Figure 1b.

The cooling sequences were extracted starting from the first non-saturated frames after the heat load (the cold frames were also subtracted from the respective sequences).

a) First setup CFRP panel, flash lamps, fast IR camera

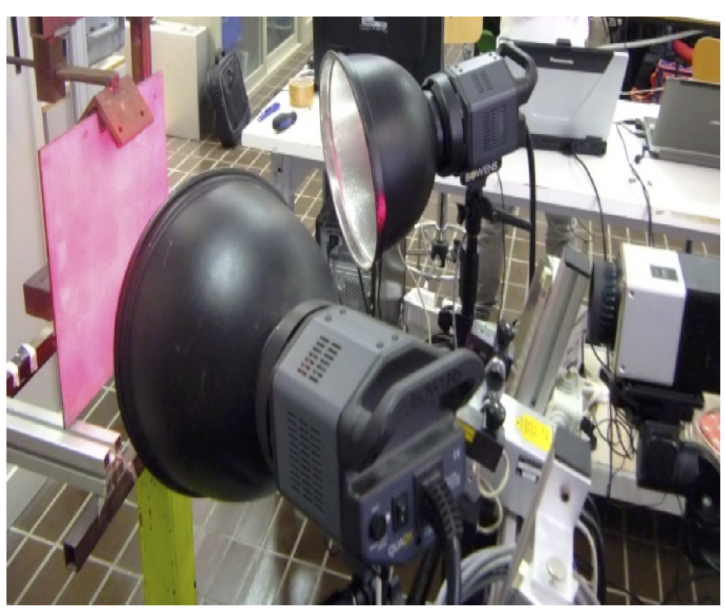

b) Second setup GRC panel, halogen lamps, slow IR camera

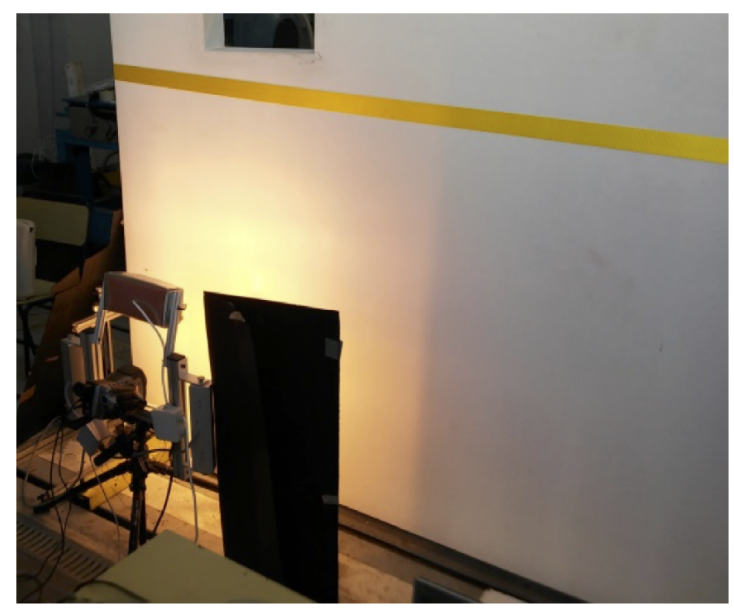

c) CFRP panel Ultrasonic $\mathrm{C}$-scan

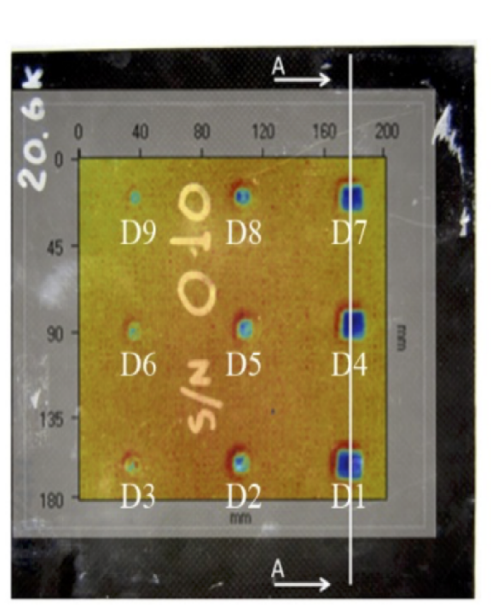

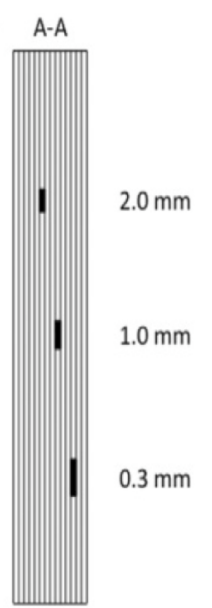

d) GRC panel
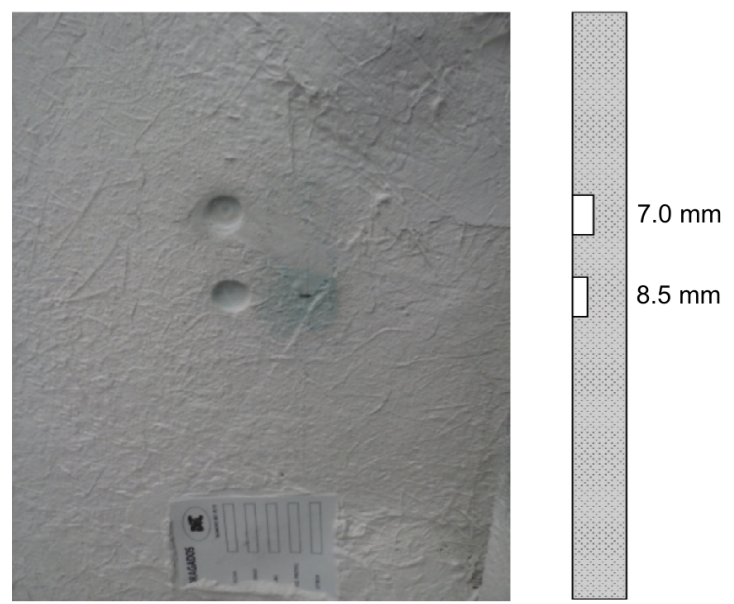

Figure 1. Experimental setups: a) CFRP panel; b) GRC panel. Specimens and defects geometries: c) CFRP panel; d) GRC panel. 


\subsection{1/qirt.2016.025}

\section{Denoising and post-processing}

The denoising approach was tested on the two test cases, so as to test its performance on thermal images gathered from materials with different thermal properties (the first specimen is characterized by high thermal diffusivity, whilst the second shows a low one). The denoising procedure consists of the following steps:

1. SWT (equations (1) to (4)) is applied to each frame $s_{0}$ of the cooling sequence, at the $L^{\text {th }}$ level. $A$ and $B$ are the high-pass and low-pass filters, respectively. The $(\cdot)$ symbol in equations (1) to (4) stands for the convolution operator.

$$
\begin{aligned}
& s_{L}=s_{0} \cdot B_{0} \cdot B_{0}^{\prime} \cdot \ldots \cdot B_{L-1} \cdot B_{L-1}^{\prime} \cdot B_{L} \cdot B_{L}^{\prime} \\
& H_{L}=s_{0} \cdot B_{0} \cdot B_{0}^{\prime} \cdot \ldots \cdot B_{L-1} \cdot B_{L-1}^{\prime} \cdot A_{L} \cdot B_{L}^{\prime} \\
& V_{L}=s_{0} \cdot B_{0} \cdot B_{0}^{\prime} \cdot \ldots \cdot B_{L-1} \cdot B_{L-1}^{\prime} \cdot B_{L} \cdot A_{L}^{\prime} \\
& D_{L}=s_{0} \cdot B_{0} \cdot B_{0}^{\prime} \cdot \ldots \cdot B_{L-1} \cdot B_{L-1}^{\prime} \cdot A_{L} \cdot A_{L}^{\prime}
\end{aligned}
$$

2. $H_{L}, V_{L}$ and $D_{L}$ are clustered using an intra-scale and inter-scale context modelling as presented in [4]. The context value $P$ for each pixel $(m, n)$ at the $L^{\text {th }}$ level is a function of the magnitude of its neighbours at levels $L$ and $L+1$ :

$$
\vec{P}_{L}(m, n)=u_{L, L+1}(m, n) \cdot h_{L}
$$

H elusters

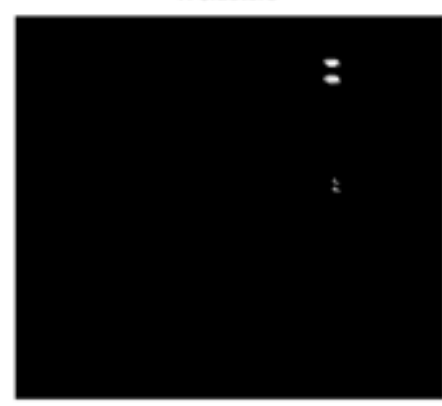

V elusters

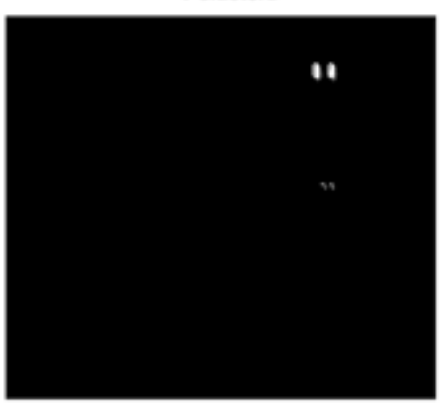

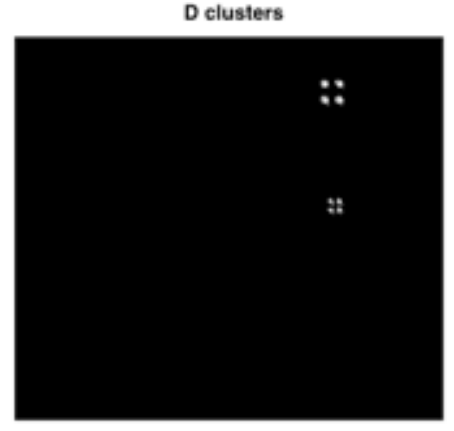

Figure 2. Context modeling.

In (5) $u_{L, L+1}$ is the neighbours list at the $L^{\text {th }}$ and $L+1^{\text {th }}$ levels and $h$ a weighting function determined by a least square estimation (more details are reported in [4]). The context value is used for grouping the pixels into a predefined number of clusters: assuming a grouping into two clusters (see Figure 2), the defects boundaries are well evident with respect to the background, as a consequence of the different topological features. The effect of clustering is found to be a probability distribution for each cluster, which is, in this case, closer to a Gaussian distribution, respect to the non-clustered coefficient. Therefore, LMMSE approaches the optimal estimator.

3. Inter-scale LMMSE [4] is calculated for each detail coefficient $C$ at the target level $L$. Assuming $s_{0}$ is corrupted with additive Gaussian noise, the estimator $\vec{x}_{C, L}$ is the following:

$$
\vec{x}_{C, L}=P_{C, L} \cdot\left(P_{C, L}+R_{C, L}\right)^{-1} \cdot \vec{\omega}_{C, L}
$$

where $\vec{\omega}_{C, L}$ is a $M \times N \times 2$ (being $M \times N$ the image resolution) matrix obtained by overlapping the correspondent detail coefficients at levels $L$ and $L+1 . P_{C, L}$ and $R_{C, L}$ are the respective inter-scale covariance matrices for $x$ and the noise propagated, with $P_{L}$ calculated for each cluster.

4. $s_{L}$ is moved into the frequency domain and high-frequency noise is removed. Then, inverse FFT is applied.

5. The denoised frame is reconstructed using low-passed $s_{L}$ and LMMSE estimators for $H_{L}, V_{L}$ and $D_{L}$.

Steps 1 to 5 are applied to each frame of the cooling sequence. Then, the denoised sequences are postprocessed with PPT. A binarization procedure is performed on the phase maps in order to highlight the presence of defects. The adaptive threshold is calculated according to the statistical distributions of the phase values at the selected frequencies, corresponding to a certain value of the correspondent cumulated probability function: $99 \%$ for the CFRP panel and $90 \%$ for the GCR panel, considering the different ratios between the sound and defect areas.

The aforementioned procedure has found to be highly dependent on the correct choice of wavelet parameters, i.e. decomposition level and wavelet basis. These have to be optimized on the frame that carries most of the defect content in the raw thermal sequence (best thermal image). Authors propose to identify the optimal decomposition level $L^{*}$ as the one where the standard deviations std of the wavelet coefficients ( $\sigma_{H L}, \sigma_{V L}$ and $\sigma_{D L}$ in equations (7) to (9)) become greater than the standard deviations of the noise propagated on the same coefficients ( $\sigma_{H L}$ noise, $\sigma_{V L}$ noise and $\sigma_{D L_{-}}$noise in equations (10) to (12).

$$
\begin{aligned}
& \sigma_{H_{L}}=\operatorname{std}\left(H_{L}\right) \\
& \sigma_{V_{L}}=\operatorname{std}\left(V_{L}\right)
\end{aligned}
$$




$$
\begin{aligned}
& \sigma_{D_{L}}=\operatorname{std}\left(D_{L}\right) \\
& \sigma_{H_{L-} \text { noise }}=\sigma_{0} \cdot B_{0} \cdot B_{0}^{\prime} \cdot \ldots \cdot B_{L-1} \cdot B_{L-1}^{\prime} \cdot A_{L} \cdot B_{L}^{\prime} \\
& \sigma_{V_{L-} \text { noise }}=\sigma_{0} \cdot B_{0} \cdot B_{0}^{\prime} \cdot \ldots \cdot B_{L-1} \cdot B_{L-1}^{\prime} \cdot B_{L} \cdot A_{L}^{\prime} \\
& \sigma_{D_{L-} \text { noise }}=\sigma_{0} \cdot B_{0} \cdot B_{0}^{\prime} \cdot \ldots \cdot B_{L-1} \cdot B_{L-1}^{\prime} \cdot A_{L} \cdot A_{L}^{\prime}
\end{aligned}
$$

In equations (10) to (12) $\sigma_{0}$ denotes the standard deviation of the noise affecting the original frame, and it is calculated as the standard deviation of the difference between two consecutive frames. This is expected to ensure the maximum noise removal without any loss of significant information. The optimal basis is selected according to the performance index $r$ proposed in [4] and reported in (13) for ease of readability.

$r=M \cdot e^{-E}$

In (13) $M$ is the mutual information of the noisy coefficient and its estimator $x_{L}$, which is calculated according to KullbackLeibler measure as the distance between the joint distribution $p\left(C_{L}, x_{L}\right)$ and the product of marginal distributions $p\left(C_{L}\right)$. $p\left(x_{L}\right) . E$ is the Hellinger distance between the real joint probability density function $p\left(x_{L}, x_{L+1}\right)$ and the correspondent Gaussian probability density function.

Several wavelet families have been tested (Haar, Daubechies, Symlets, Biortogonal) at various decomposition levels (1 to 5). Applying the optimal level selection criterion presented before, the fourth level was found to be the optimal one (Figure 3) for both sequences (CFRP panel and GRC panel). bior5.5 (red bar in Figure 3) is the wavelet basis which maximises the performance index $r$ (Figure 3 ) and the Energy-to-Shannon-Entropy Ratio for both sequences.
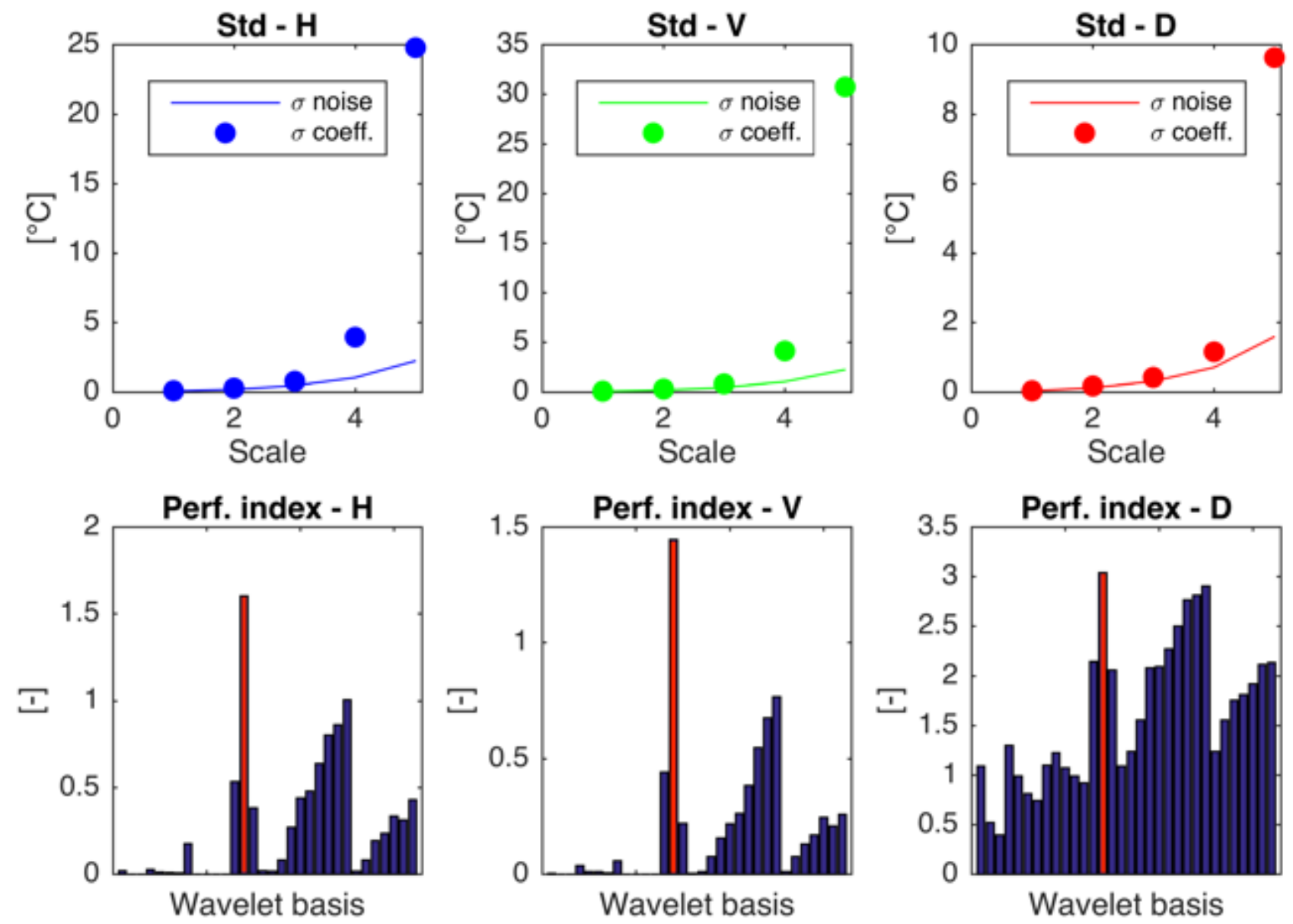

Figure 3. Decomposition level and wavelet basis optimization - CFRP panel.

\section{Results}

Small-size and high-depth defects are characterized by a low thermal contrast with respect to the background. This aspect prevents their detection directly on the raw thermal images. Further, when binarization is applied directly on the thermal images, the presence of hot areas due to an inhomogeneous heating resulted in the enhancement of spots 
not corresponding to real defects, as it can be seen in Figure 4. The problem is more evident in the GRC panel, because the low emissivity produces a pattern associated with the reflections of the experimental setup.

\section{Best thermal image CFRP panel}
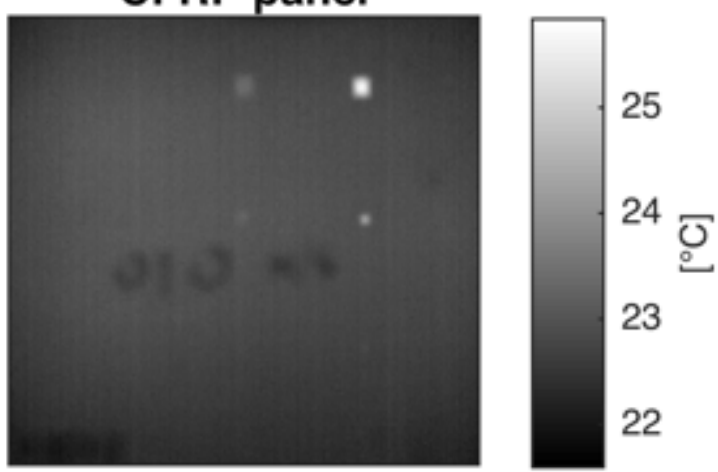

\section{Best thermal image GRC panel}

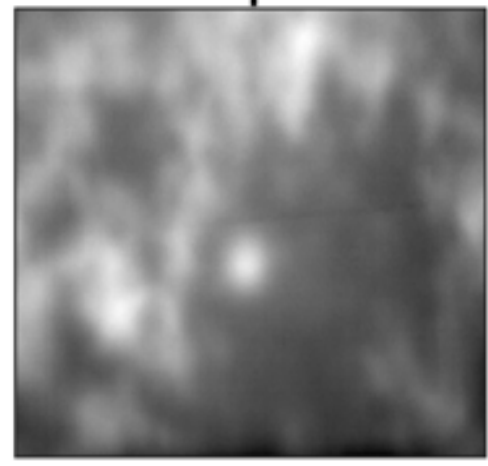

38

36

34

32

30
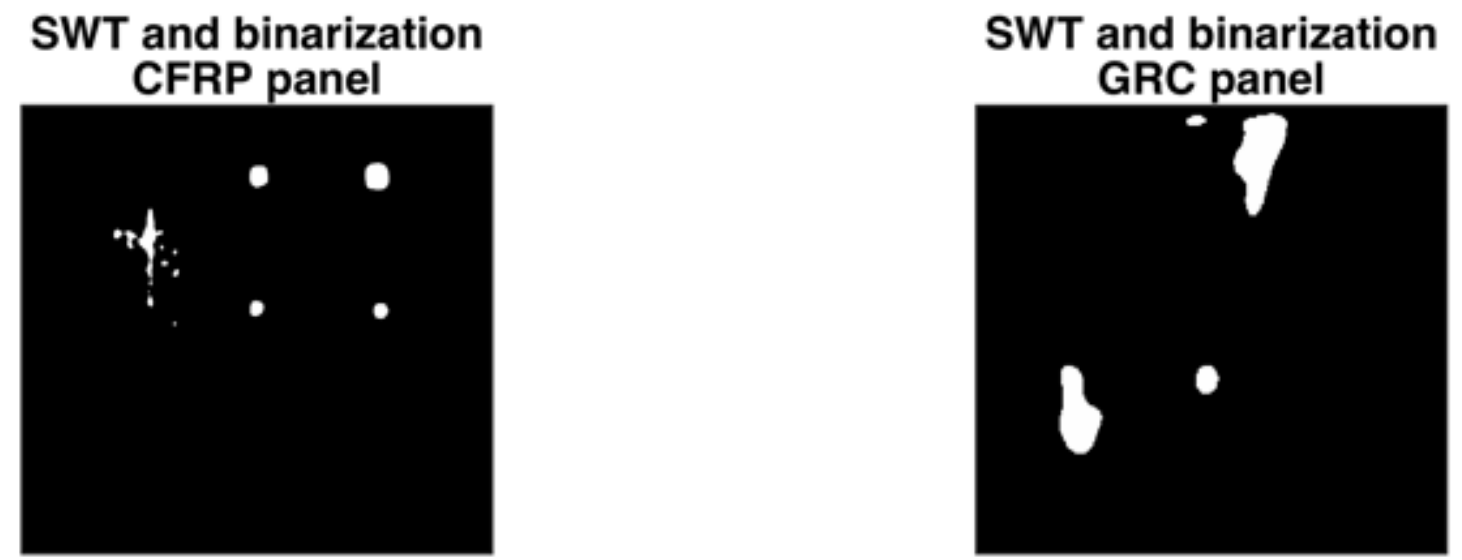

Figure 4. Best thermal images; SWT and binarization (black and white) on best thermal images.

These drawbacks were only partially overcome by PPT. As it can be seen in Figure 5 (the pattern located on the centre is due to a surface mark and should be ignored), phase maps show a flat background, which is less affected by the local distribution of the heat load and the surface emissivity. Therefore, when thresholding is applied on phase maps, the correspondent binarization is more selective with respect to the real defects. Nevertheless, high noise still affects phase maps, even at low frequency. Therefore, small-size and high-depth defects are not detected, after binarization.

The application of the SWT-denoising procedure as a pre-processing step on the raw data drastically improved the performances of PPT, once the decomposition level and wavelet basis were optimized according to the criteria discussed in Section 3. Moreover, reconstructing the original images using LMMSE estimators preserved high-frequency information, whilst noise content was abated. This resulted in a precise reconstruction of defects edges and other small details. The effects on defect detection can be well appreciated after the binarization step. On this regard, it is evident that thresholding introduces some arbitrariness; however, it was found that the thresholds indicated in Section 3 represent a good compromise between defect enhancement and noise abatement. Figure 6 is referred to the CFRP panel, and shows phase maps binarized, after a denoising with bior5.5 at levels 3 to 5 , with 4 being the optimal level. As it can be observed, at level 4 small-size and high-depth defects are detected. Besides, topological features of low and medium-depth defects are well reproduced. Indeed, sizes and shapes are coherent with those obtained from a reference ultrasonic C-scan (Figure 1). The impact of the denoising step on the defect detection capability was quantified using the CNR criterion. The CNR was calculated on the phase maps before binarization. The formula for calculating the CNR is reported in (14):

$$
C N R=\frac{\mu_{\text {kernel }}-\mu_{\text {back }}}{\left(w_{\text {kernel }} \cdot \sigma_{\text {kernel }}^{2}+w_{\text {back }} \cdot \sigma_{\text {back }}^{2}\right)^{\frac{1}{2}}}
$$

Eq. 14 requires the definition of a Region Of Interest (ROI), which covers the defect for the whole extension. Inside the ROI it is defined a kernel, whose size and shape are those of the defect. $\mu_{k e r n e l}$ is the average of the intensities inside the kernel, whilst $\sigma_{\text {kernel }}$ is the standard deviation of the intensities inside the kernel. Analogously, $\mu_{\text {back }}$ and $\sigma_{\text {back }}$ are the average and the standard deviation of the intensities inside the back, respectively, with the back defined as the 
difference between the ROI and the kernel. $w_{\text {kernel }}$ and $w_{\text {back }}$ are obtained by dividing the areas of the kernel and the back, respectively, by the total area of the ROI. Eq. (14) is implemented for each position of the kernel inside the ROI, thus it is obtained a distribution of the CNRs. The CNR of the defect is defined as the maximum of that distribution. The geometry of the kernel was obtained from the ultrasonic C-scan. An in-depth discussion about the CNR criterion is reported in [7].

As it can be observed looking at the CNR values reported in Table 1, denoising is effective in improving the defects contrast whilst reducing noise, as long as the decomposition level lies within the optimal level. However, when wavelet decomposition overcomes the optimal level, the denoising procedure results in worsening effects and several defects are not detectable anymore.

Phase

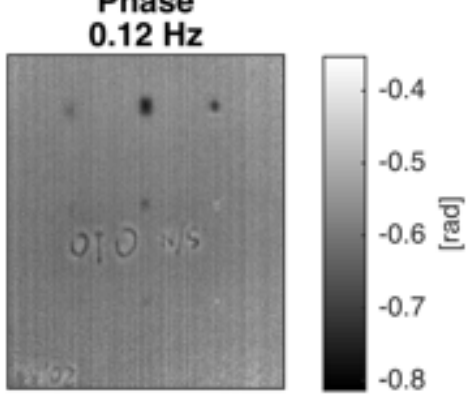

Phase binarized $0.12 \mathrm{~Hz}$

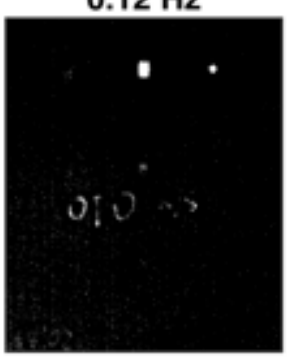

Phase

\section{$0.18 \mathrm{~Hz}$}

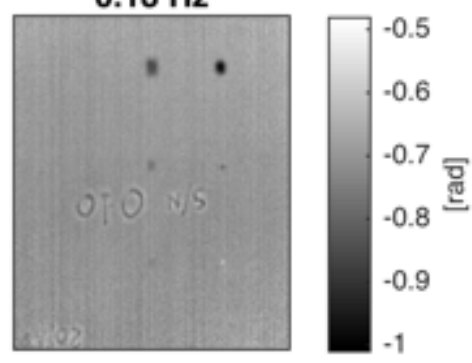

\section{Phase binarized \\ $0.18 \mathrm{~Hz}$}

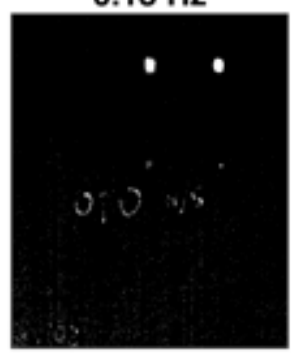

Phase

$0.72 \mathrm{~Hz}$

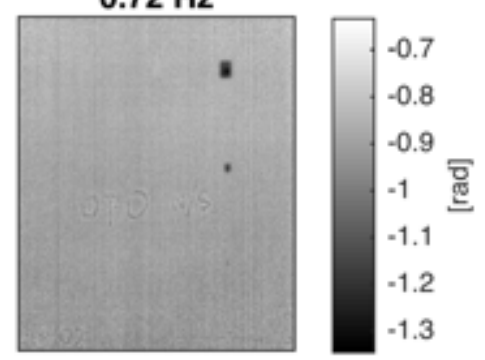

Figure 5. PPT on CFRP panel. Phase maps before and after binarization (black and white). No denoising.

\begin{tabular}{|c|c|c|c|c|}
\hline & No denoising & Level 3 & Level 4 & Level 5 \\
\hline Big-size, low-depth defect & 5.9 & 7.9 & 7.9 & 3.7 \\
\hline Medium-size, low-depth defect & 4.9 & 10.0 & 10.3 & 3.1 \\
\hline Small-size, low-depth defect & 5.0 & 9.4 & 9.6 & Not detected \\
\hline Big-size, medium-depth defect & 4.1 & 7.2 & 7.3 & 3.9 \\
\hline Medium-size, medium-depth defect & 2.3 & 5.5 & 5.6 & 2.8 \\
\hline Small-size, medium-depth defect & 6.0 & 10.6 & 10.7 & Not detected \\
\hline Big-size, high-depth defect & 1.2 & 3.8 & 3.9 & 2.9 \\
\hline Medium-size, high-depth defect & 2.1 & 2.6 & 2.6 & Not detected \\
\hline Small-size, high-depth defect & Not detected & Not detected & Not detected & Not detected \\
\hline
\end{tabular}

Table 1. Contrast Noise Ratios (adimensional units, linear scale) - CFRP panel. 

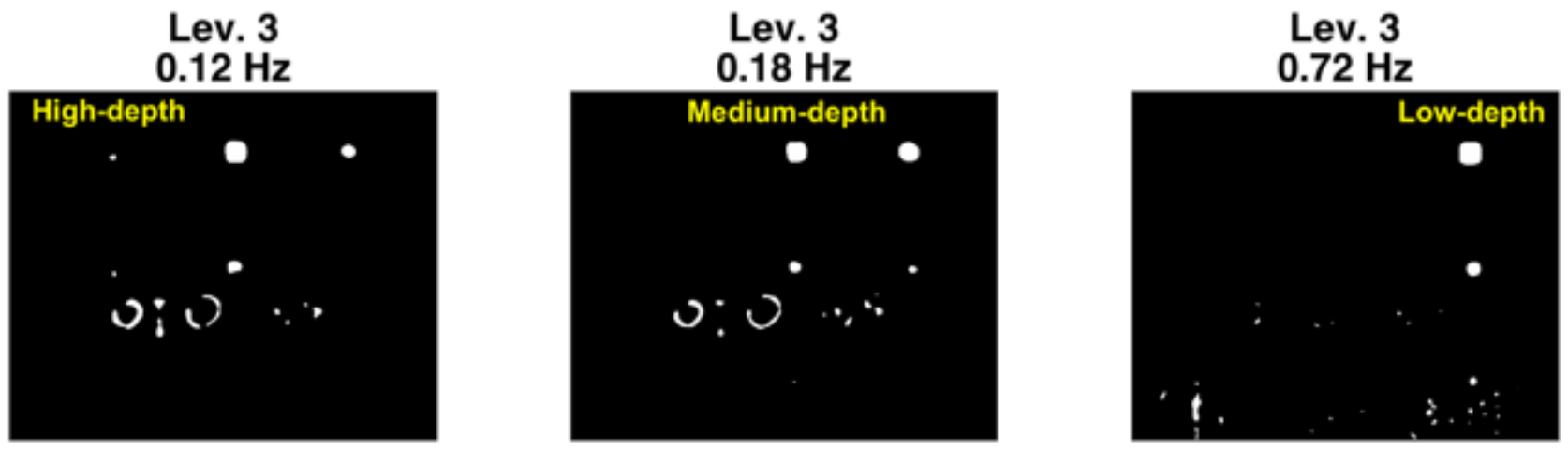

Lev. 4

$0.12 \mathrm{~Hz}$

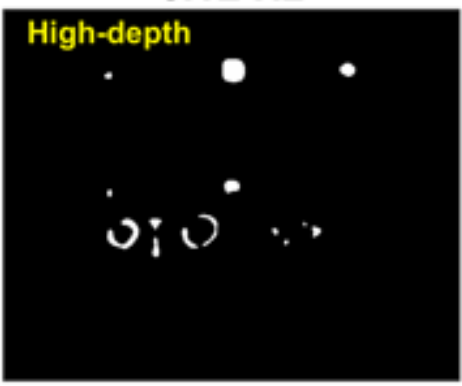

Lev. 5

$0.12 \mathrm{~Hz}$

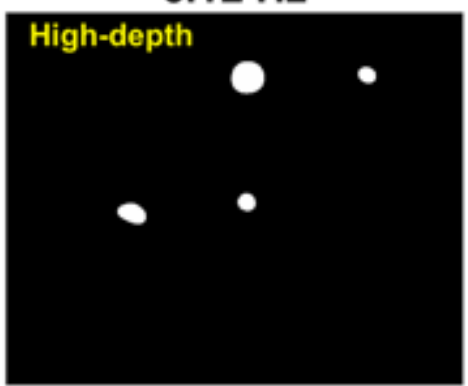

Lev. 4

$0.18 \mathrm{~Hz}$

Medium-depth

$\mathrm{O}$

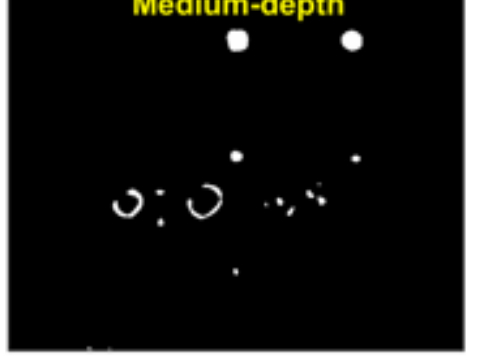

Lev. 5

$0.18 \mathrm{~Hz}$

Medium-depth

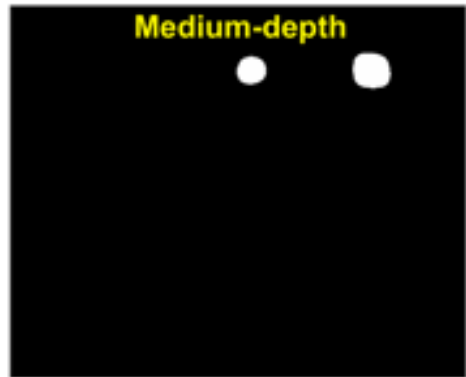

Lev. 4

$0.72 \mathrm{~Hz}$

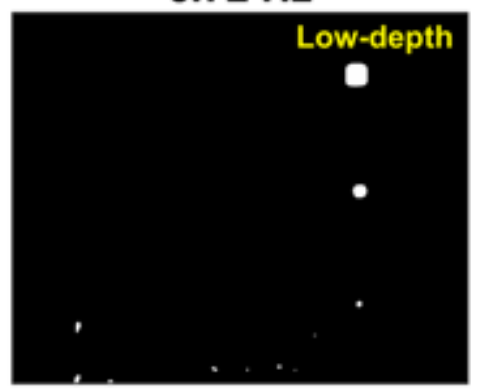

Lev. 5

$0.72 \mathrm{~Hz}$

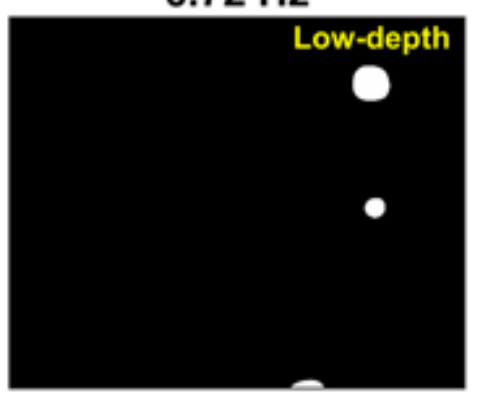

Figure 6. CFRP panel. Denoising, PPT and binarization (black and white).

When the same denoising procedure is tested on the second sequence (GRC panel), results are analogous. Figure 7 compares the phase maps at a frequency of $0.014 \mathrm{~Hz}$, obtained after denoising at the optimal (4) and sub-optimal ( 3 and 5) decomposition levels. When the decomposition level is higher then the optimal one, a relevant blurring occurs, thus preventing the detection of the high-depth defect. CNR values reported in Table 2 are in line with this observation. 

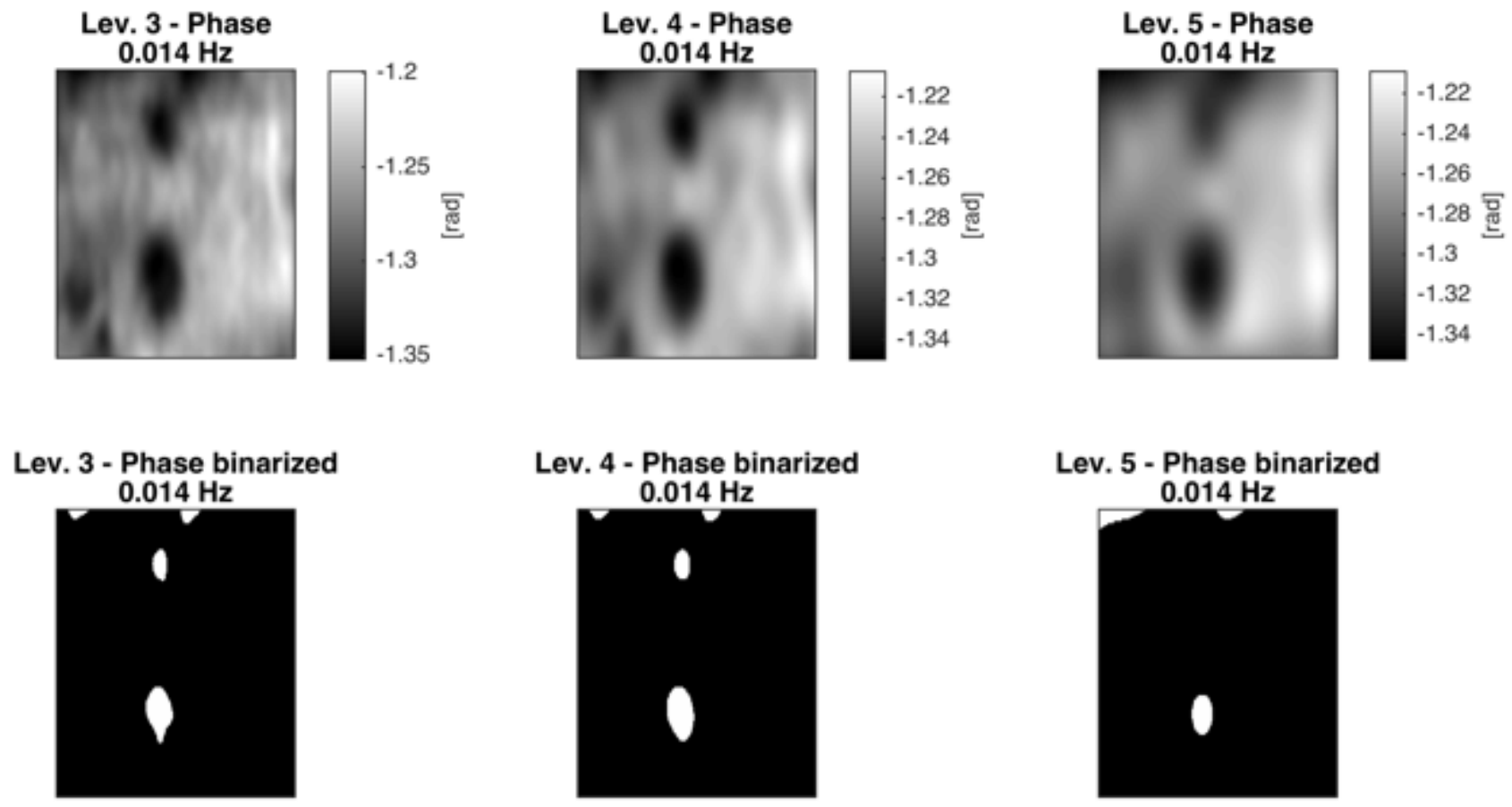

Figure 7. GRC panel. Denoising, PPT and binarization (black and white).

\begin{tabular}{|c|c|c|c|c|}
\hline & No denoising & Level 3 & Level 4 & Level 5 \\
\hline Low-depth defect & 2.2 & 2.4 & 2.5 & 2.4 \\
\hline High-depth defect & 1.6 & 1.8 & 1.8 & Not detected \\
\hline
\end{tabular}

\section{Conclusions}

A denoising procedure based on SWT, LMMSE and noise filtering in the frequency domain was applied to results of PT tests. An innovative criterion was proposed for selecting the optimal decomposition level. The samples tested were two panels of different materials (CFRP and GRC) with defects of various sizes and depths. Denoised sequences were post-processed using PPT and binarization.

The denoising approach demonstrated its capability in providing marked enhancement in terms of defects detection, once the decomposition level and the wavelet basis have been optimized. CNRs showed improvements in the order of $80 \%$ for the CFRP panel and $11 \%$ for the GRC panel. Using statistical estimators is effective in producing strong noise abatement, whilst preserving high-frequency information. As a consequence, topological features like defects edges are quite well reproduced, even for small-size defects. The optimal level selection criterion proposed was found to be relevant for ensuring the maintenance of such features.

\section{Acknowledgements}

This work was supported by the EU Project INSITER (Intuitive Self-Inspection Techniques using Augmented Reality for construction, refurbishment and maintenance of energy-efficient buildings made of prefabricated components). The project has received funding from the European Union H2020 framework program under grant agreement $n^{\circ} 636063$. The authors acknowledge the INSITER partner DRAGADOS for having provided the GRC panel.

\section{REFERENCES}

[1] Wang X. H., Istepanin R. S., Song Y. H., "Micro array image enhancement by denoising using stationary wavelet transform", IEEE Transactions on Nano Bioscience, vol. 2, pp. 184-189, 2003.

[2] Revel G. M., Chiariotti P., Copertaro E., Pandarese G., "Stationary Wavelet Transform in Pulsed Thermography: influence of camera resolution on defect detection", Proceedings of $13^{\text {th }}$ Advanced Infrared Technology and Application conference, Pisa, Italy, 2015.

[3] Liu T., Zhang W., Yan S., "A novel image enhancement algorithm based on stationary wavelet transform for infrared thermography to the de-bounding defect in solid rocket motors", Mechanical Systems and Signal Processing, vol. 62-63, pp. 366-380, 2015. 


\subsection{1/qirt.2016.025}

[4] Zhang L., Bao P., "Multiscale LMMSE-Based Image Denoising With Optimal Wavelet Selection", IEEE Transactions on Circuits and Systems for Video Technology, vol. 15, pp. 469-481, 2005.

[5] Bafroui H. H., Ohadi A., "Application of wavelet energy and Shannon entropy for feature extraction in gearbox fault detection under varying speed conditions", Neurocomputing, vol. 133, pp. 437-445, 2014.

[6] "http://www.insiter-project.eu/".

[7] Revel G. M., Rocchi S., Defect detection in ceramic materials by quantitative infrared thermography, Proceedings of the $8^{\text {th }}$ Quantitative Infrared Thermography conference, 2006. 